\title{
Ultrafast intersystem crossing in xanthone from wavepacket dynamics Marc Alías-Rodríguez, ${ }^{\dagger, \ddagger}$ Coen de Graaf, ${ }^{\dagger}, \Phi$ and Miquel Huix-Rotllant ${ }^{*, \ddagger}$ \\ $\dagger$ Departament de Química Física i Inorgànica, Universitat Rovira i Virgili, Marcel-lí Domingo 1, Tarragona, 4300\%, Spain. \\ $\ddagger$ Aix-Marseille Univ, CNRS, ICR, Marseille, 13397, France. \\ ๆICREA, Passeig Lluís Companys 23, Barcelona, 08010, Spain \\ Received December 8, 2021; E-mail: miquel.huix-rotllant@cnrs.fr
}

\begin{abstract}
Most aromatic ketones containing first-row elements undergo unexpectedly fast intersystem crossing in few tens of picoseconds and a quantum yield close to unity. Among them, xanthone (9H-xanthen-9-one) possesses one of the fastest singlet-triplet rates of $\sim 1.5 \mathrm{ps}$. The exact mechanism of this unusually fast transition is still under debate. Here, we perform the wavepacket dynamics of the photochemistry of xanthone in the gas phase and in polar solvents. We show that xanthone follows El-Sayed's rule for intersystem crossing. From the second singlet excited state, the mechanism is sequential: (i) an internal conversion between singlets ${ }^{1} \pi \pi^{*} \rightarrow{ }^{1} n \pi^{*}$ (85 fs), (ii) an intersystem crossing ${ }^{1} n \pi^{*} \rightarrow{ }^{3} \pi \pi^{*}(2.0 \mathrm{ps})$, and (iii) an internal conversion between triplets ${ }^{3} \pi \pi^{*} \rightarrow{ }^{3} n \pi^{*}$ (602 fs). Each transfer finds its origin in a barrierless access to electronic state intersections. These intersections are close to minimum energy structures, allowing for efficient transitions from the initial singlet state to the triplets.
\end{abstract}

Aromatic ketones are well known for their phosphorescent properties due to an efficient intersystem crossing (ISC) ${ }^{1-4}$ non-radiative transfer between singlet and triplet excited states of $n \pi^{*}$ and $\pi \pi^{*}$ character. The $n$ orbital corresponds essentially to carbonyl's lone pair orbital, while the $\pi$ and $\pi^{*}$ are a mixture of bonding and anti-bonding combinations of the atomic $\mathrm{p}_{z}$ orbitals centred on carbonyl and aromatic rings. According to photochemistry rules (El-Sayed's), ${ }^{5}$ an efficient ISC is characterized by simultaneous strong spinorbit coupling and a small energetic singlet-triplet gap for states of dissimilar character, for example ${ }^{1} n \pi^{*} \rightarrow{ }^{3} \pi \pi^{*}$ or ${ }^{1} \pi \pi^{*} \rightarrow{ }^{3} n \pi^{*}$. The lifetime of triplet states in aromatic ketones are reported to last from few to several tens of picoseconds, depending mainly on the energetic gap between $n \pi^{*}$ and $\pi \pi^{*}$ states. For example, acetophenone ISC rate is reported to be $42 \mathrm{ps},{ }^{6}$ benzophenone $5-10 \mathrm{ps},{ }^{7}$ anthrone $70 \mathrm{ps},{ }^{8}$ etc. The singlet-triplet gap can be modulated and states can be interchanged depending on the type of ketone,${ }^{8-11}$ solvent polarity, ${ }^{12-16}$ temperature, ${ }^{2}$ pressure,${ }^{17}$ substituents, ${ }^{16,18}$ concentration, ${ }^{19}$ etc.

It is widely accepted that vibrations play a central role in modulating the efficiency of non-radiative decays, ${ }^{20}$ and ISC are no exception. ${ }^{21-24}$ One of us performed wavepacket dynamics simulations in acetophenone, showing a rapid ${ }^{3} n \pi^{*} /{ }^{3} \pi \pi^{*}$ interconversion after the ${ }^{1} n \pi^{*} \rightarrow{ }^{3} \pi \pi^{*}$ ISC. ${ }^{23}$ Granucci and coworkers performed a study of benzophenone using fewest-switches surface hopping, obtaining a 16 ps direct transfer from the lowest singlet to the lowest triplet, due to the mixed $n \pi^{*}$ and $\pi \pi^{*}$ character of the states. ${ }^{24}$ Marian and coworkers have studied the ISC of thioxanthone ${ }^{25-27}$ and xanthone ${ }^{22}$ using Fermi's golden rule to determine vi- bronic ISC rates.

The triplet quantum yield of xanthone in solution is close to unity. ${ }^{12}$ Among the aromatic ketones, xanthone has one of the fastest ISC, estimated to be around 1-1.5 picoseconds in ethanol by femtosecond fluorescent experiments. ${ }^{28-30}$ In these experiments, two kinetic steps of 1.5 ps and 12 ps were obtained. Two mechanisms were proposed to explain these rates: (i) sequential mechanism, with a fast ${ }^{1} \pi \pi^{*} \rightarrow{ }^{3} n \pi^{*}$ transfer in 1.5 ps and a slow component of 12 ps belonging to the internal conversion between triplets, and (ii) a branching mechanism, in which the $1.5 \mathrm{ps}$ component corresponds to both the ISC ${ }^{1} \pi \pi^{*} \rightarrow{ }^{3} n \pi^{*}$ and the internal conversion ${ }^{1} \pi \pi^{*} \rightarrow{ }^{1} n \pi^{*}$, and the slow $12 \mathrm{ps}$ component to the ${ }^{1} n \pi^{*} \rightarrow{ }^{3} \pi \pi^{*}$ transfer from the lowest singlet. RaiConstapel and coworkers simulated the ISC rate in gas phase and solution of xanthone using Fermi's golden rule. ${ }^{22}$ In the gas phase, they obtained a fast ${ }^{1} \pi \pi^{*} \rightarrow{ }^{3} \pi \pi^{*}$ ISC of about 5 ps, and a ${ }^{1} \pi \pi^{*} \rightarrow{ }^{3} n \pi^{*}$ ISC of $200 \mathrm{ps}$, in apparent contradiction with El-Sayed's rule. As for the ${ }^{1} n \pi^{*} \rightarrow{ }^{3} \pi \pi^{*}$ ISC rate of $0.13 \mathrm{ps}$, which is $10-100$ times faster than the experimental reported values. The ${ }^{1} n \pi^{*} \rightarrow{ }^{3} n \pi^{*}$ transition was estimated at ca. 330 ps. Recently, Bracker et al. extended the use of Fermi's golden rule for both the internal coversion and the ISC. ${ }^{31}$ Their results suggested that ${ }^{1} \pi \pi^{*}{ }^{3} \pi \pi^{*}$ ISC and ${ }^{1} \pi \pi^{*}{ }_{-}^{1} \mathrm{n} \pi^{*}$ IC are competitive processes from which a branched mechanism was infered.

Here, we report the wavepacket dynamics of xanthone including the lowest two singlet and four triplet excited states. The model contains all non-adiabatic and spin-orbit couplings to represent the internal conversion and ISC on the same footing, including the effect of Duschinsky rotations. ${ }^{32}$ The total vibronic Hamiltonian can be written as

$$
\mathbf{H}(\mathbf{Q})=[\mathbf{T}(\mathbf{Q})+\mathbf{V}(\mathbf{Q})] \mathbf{1}+\mathbf{H}^{\mathrm{NA}}(\mathbf{Q})+\mathbf{H}^{\mathrm{SO}}(\mathbf{Q}) .
$$

Here, $\mathbf{T}(\mathbf{Q})$ and $\mathbf{V}(\mathbf{Q})$ are the kinetic and diabatic potential energies, and $\mathbf{H}^{\mathrm{NA}}(\mathbf{Q})$ and $\mathbf{H}^{\mathrm{SO}}(\mathbf{Q})$ are the vibronic non-adiabatic and spin-orbit couplings. The explicit expressions for the Hamiltonian blocks and the parametrization are described in Sec. S1 of the supporting information (SI).

Xanthone lowest energy state is a singlet $1^{1} \mathrm{~A}_{1}$ state (using $\mathrm{C}_{2 v}$ point group symmetry labeling, with the orientation as described in Fig. 1). At this geometry, the $1^{1} \mathrm{~A}_{2}$ state is the first excited singlet, corresponding to a $19 \mathrm{~b}_{2} \rightarrow 6 \mathrm{~b}_{1}$ transition (also called ${ }^{1} n \pi^{*}$ state), which is dipole-forbidden. The second singlet excited state is the $2^{1} \mathrm{~A}_{1}$ state, which is represented by a $5 \mathrm{~b}_{1} \rightarrow 6 \mathrm{~b}_{1}$ transition (more commonly referred to ${ }^{1} \pi \pi^{*}$ state), and is dipole-allowed. In the minimum energy structure of $1^{1} \mathrm{~A}_{1}$ state (Fig. 1), the triplets $1^{3} \mathrm{~A}_{2}$ and $1^{3} \mathrm{~A}_{1}$ are quasi-degenerate ( $0.03 \mathrm{eV}$ gap), $0.166 \mathrm{eV}$ below the singlet $1^{1} \mathrm{~A}_{2}$ state, in good agreement with the experimental value of $0.14 \mathrm{eV} .^{33}$ The $1^{1} \mathrm{~A}_{2}$ state is only $0.06 \mathrm{eV}$ below 
(a)

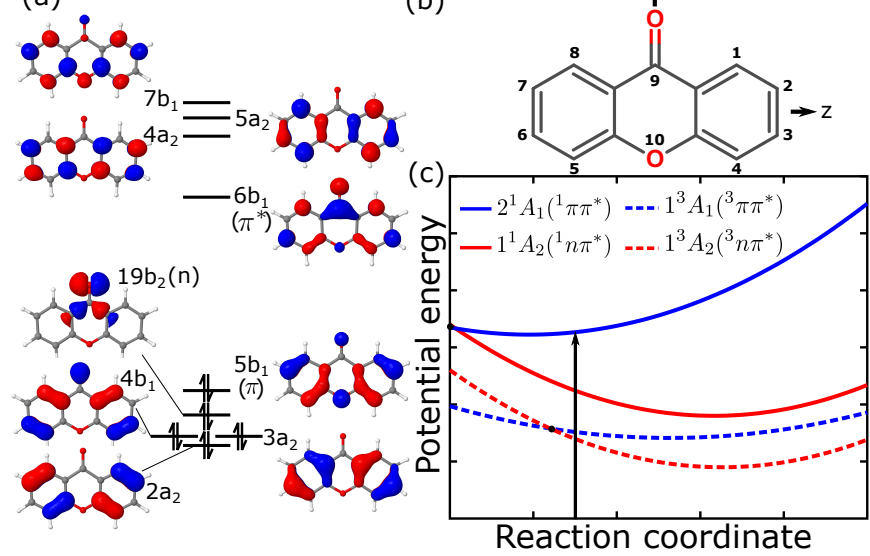

Figure 1. (a) the 5 highest occupied and 4 lowest unoccupied orbitals with $\mathrm{C}_{2 v}$ labels and common name in parenthesis. (b) structure of 9H-xanthen-9-one (xanthone) with number and Cartesian axis orientation. (c) schematic diabatic potential energy curve of the two lowest singlet (solid line) and triplet (dashed line) excited states of $n \pi^{*}$ (red) and $\pi \pi^{*}$ (blue) character. The vertical arrow indicates the absorption from the ${ }^{1} \pi \pi$ ground state (not shown in the figure).

the $2^{3} \mathrm{~A}_{1}$ described mainly by a $4 \mathrm{~b}_{1} \rightarrow 6 \mathrm{~b}_{1}$ transition, and the triplet $1^{3} \mathrm{~B}_{2}$ described mainly by a $3 \mathrm{a}_{2} \rightarrow 6 \mathrm{~b}_{1}$ is found $\sim 0.11 \mathrm{eV}$ above $1^{1} \mathrm{~A}_{2}$. The $2^{1} \mathrm{~A}_{1}$ state $\left(5 \mathrm{~b}_{1} \rightarrow 6 \mathrm{~b}_{1}\right)$ is the highest energy state, $0.4 \mathrm{eV}$ above the $1^{1} \mathrm{~A}_{2}$ state. Minimal energy structures of the lowest singlet and triplet states are planar of quasi- $\mathrm{C}_{2 v}$ symmetry. As for the state crossings, on the one hand the $2^{1} \mathrm{~A}_{1} / 1^{1} \mathrm{~A}_{2}\left({ }^{1} \pi \pi^{*} /{ }^{1} n \pi^{*}\right)$ cross in an accidental different symmetry structure of $\mathrm{C}_{s}$ resulting from the linear combination of the carbonyl symmetric stretching (essentially of $\mathrm{a}_{1}$ symmetry) and an asymmetric stretching vibration of mostly $b_{1}$ symmetry involving the carbon atoms on the aromatic ring. On the other hand, $1^{1} \mathrm{~A}_{2}$ crosses with $1^{3} \mathrm{~A}_{1}$ state in a quasi- $\mathrm{C}_{2 v}$ structure expanded through totally symmetric vibrations involving the carbonyl symmetric stretching of the aromatic rings.

The dynamic evolution of diabatic populations is shown in Fig. 2. The simulations started at the $2^{1} \mathrm{~A}_{1}$ state. The population rapidly decays in the first $200 \mathrm{fs}$, concomitant to the $1^{1} \mathrm{~A}_{2}$ population rise, indication of a fast internal conversion. This is due to the barrierless access to two $1^{1} \mathrm{~A}_{2} / 2^{1} \mathrm{~A}_{1}$ accidental different symmetry intersection. In this process, $80 \%$ of the $2^{1} \mathrm{~A}_{1}$ is transferred to $1^{1} \mathrm{~A}_{2}$, and $20 \%$ of the population remains trapped in $2^{1} \mathrm{~A}_{1}$ decaying at a slower rate. The $1^{1} \mathrm{~A}_{2}$ being the major state after $200 \mathrm{fs}$ rapidly decays to the triplets. Initially, the ISC is mainly due to $1^{1} \mathrm{~A}_{2} \rightarrow 1^{3} \mathrm{~A}_{1}$ but also $1^{1} \mathrm{~A}_{2} \rightarrow 2^{3} \mathrm{~A}_{1}$. These are the main channels for generating triplet population, consistent thus with El-Sayed's rule for ISC. The transfer between triplets slowly builds up the population on the lowest triplet $1^{3} \mathrm{~A}_{2}$.

A first-order kinetic mechanism can be extracted from the 1.5 ps population dynamics of Fig. 2 (see Fig. S7 in SI). From the model, we can infer that the mechanism follows mainly a sequential transfer: $2^{1} \mathrm{~A}_{1} \stackrel{\mathrm{IC}}{\longrightarrow} 1^{1} \mathrm{~A}_{2} \stackrel{\mathrm{ISC}}{\longrightarrow} 1^{3} \mathrm{~A}_{1} \stackrel{\mathrm{IC}}{\longrightarrow}$ $1^{3} \mathrm{~A}_{2}$. First, the $2^{1} \mathrm{~A}_{1}$ state decays biexponentially to the $1^{1} \mathrm{~A}_{2}$ state via internal conversion. About $80 \%$ of the population decays in a fast rate of $85 \mathrm{fs}$. This fast component is explained as the first time the wavepacket reaches the $2^{1} \mathrm{~A}_{1} / 1^{1} \mathrm{~A}_{2}$ crossing with a large momentum, since the path to it is barrierless. Only activation of coupling modes of $a_{2}$ symmetry can make this transition happen, which

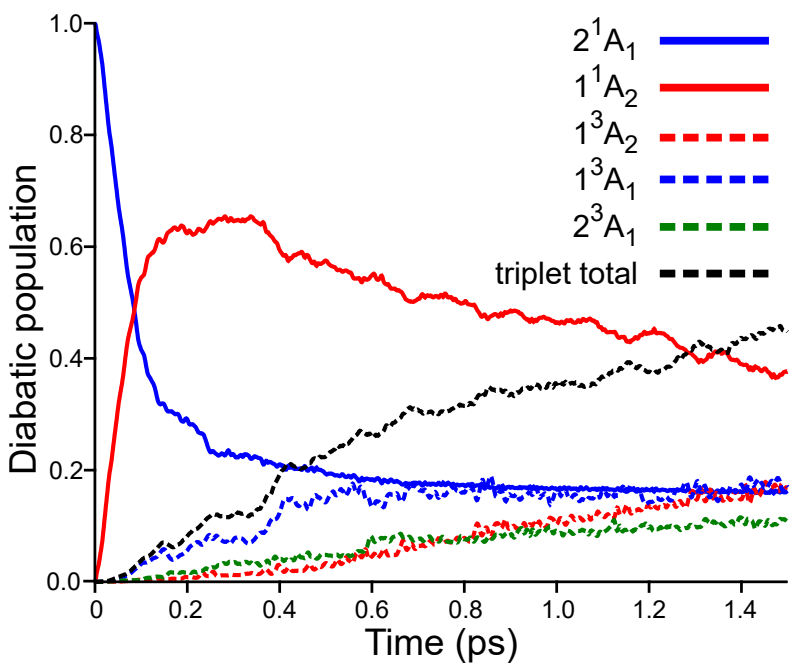

Figure 2. Diabatic population evolution for the singlets $2^{1} \mathrm{~A}_{1}$ $\left({ }^{1} \pi \pi^{*}\right.$, solid blue) and $1^{1} \mathrm{~A}_{2}\left({ }^{1} n \pi^{*}\right.$, solid red), and triplets $1^{3} \mathrm{~A}_{2}$ $\left({ }^{3} n \pi^{*}\right.$, dashed red), $1^{3} \mathrm{~A}_{1}\left({ }^{3} \pi \pi^{*}\right.$, dashed blue) and $2^{3} \mathrm{~A}_{1}$ (dashed green). Black dashed line corresponds to the sum of all triplet populations. The dynamics have started from $2^{1} \mathrm{~A}_{1}$.

could explain the incomplete transfer. After that, $15 \%$ of the wavepacket remains trapped in the $2^{1} \mathrm{~A}_{1}$ minimum, and slowly decays to $1^{1} \mathrm{~A}_{2}$ with a rate of $5 \mathrm{ps}$ via the activation of coupling modes. In the $1^{1} \mathrm{~A}_{2}$ state, there are three possible ISC transfers. The $1^{1} \mathrm{~A}_{2} \rightarrow 2^{3} \mathrm{~A}_{1}$ and $1^{1} \mathrm{~A}_{2} \rightarrow 1^{3} \mathrm{~B}_{2}$ triplet rates are obtained at 6.73 and 44.64 respectively ps. In this time, we do not observe a fast internal conversion to the $1^{3} \mathrm{~A}_{1}$ and $1^{3} \mathrm{~A}_{2}$, but probably this occurs at longer times. The fastest important ISC is the $1^{1} \mathrm{~A}_{2} \rightarrow 1^{3} \mathrm{~A}_{1}$, which is the first populated triplet in time. The rate for this transfer is given by $2.0 \mathrm{ps}$, which is probably explained by a strong spin-orbit coupling and the fact that the ISC minimum energy crossing geometry is close to the minimum of $1^{1} \mathrm{~A}_{2}$ state. The internal conversion between $1^{3} \mathrm{~A}_{1} \leftrightarrow 1^{3} \mathrm{~A}_{2}$ is the only pathway we obtained of population of the lowest triplet state, and is estimated at $602 \mathrm{fs}$ for the transfer to $1^{3} \mathrm{~A}_{2}$ and $803 \mathrm{fs}$ for the back transfer to $1^{3} \mathrm{~A}_{1}$.

In the literature, a major channel corresponding to a "nonKasha" transfer directly from $2^{1} \mathrm{~A}_{1}$ to the $1^{3} \mathrm{~A}_{2}$ triplet was evoked, first by Baba and coworkers for xanthone in the gas phase, ${ }^{33,34}$ and later confirmed by Gilch and coworkers for xanthone in ethanol. ${ }^{28,29}$ Baba et al. estimated the rate of transfer from $2^{1} \mathrm{~A}_{1}$ in the gas phase at ca. $100 \mathrm{fs},{ }^{34}$ while the experiments of Gilch et al. in ethanol estimated it at 1.5 ps. ${ }^{28}$ Recently, theoretical studies using rate theory in the gas phase by Rai-Constapel and coworkers predicted a 5 ps for the $2^{1} \mathrm{~A}_{1} \rightarrow 1^{3} \mathrm{~A}_{1}$ (El-Sayed's forbidden), while the $2^{1} \mathrm{~A}_{1} \rightarrow 1^{3} \mathrm{~A}_{2}$ (El-Sayed's allowed) was found having a $200 \mathrm{ps}$ rate. ${ }^{22}$ In the gas phase, the main decay channel observed from $2^{1} \mathrm{~A}_{1}$ is to the $1^{1} \mathrm{~A}_{2}$. This is estimated at a rate of $135 \mathrm{fs}$, close to the experimental decay of $100 \mathrm{fs}$ observed for $2^{1} \mathrm{~A}_{1}$ in the gas phase. ${ }^{34}$ The $2^{1} \mathrm{~A}_{1}$ state has an almost negligible vibronic spin-orbit coupling with energetically close $2^{3} \mathrm{~A}_{1}$ and $1^{3} \mathrm{~B}_{2}$ states (see Table $\mathrm{S} 7$ in SI). The $2^{3} \mathrm{~A}_{1}$ triplet receives $5-10 \%$ population, while the population to the $1^{3} \mathrm{~B}_{2}$ states is negligible. This could explain the triplet coupling evoked for the $2^{3} \mathrm{~A}_{1}$ state. ${ }^{33}$ No direct transfer from $2^{1} \mathrm{~A}_{1}$ is observed to $1^{3} \mathrm{~A}_{2}$ nor $1^{3} \mathrm{~A}_{1}$ states. Rather, the ISC channel is $1^{1} \mathrm{~A}_{2} \rightarrow 1^{3} \mathrm{~A}_{1}$ in a rate of $2.0 \mathrm{ps}, 10$ times slower than the rate obtained by Rai-Constapel and coworkers. ${ }^{22}$ The combined $1^{1} \mathrm{~A}_{2} \rightarrow$ triplets leads to a rate transfer of $1.5 \mathrm{ps}$. A 
similar rate is obtained if the dynamics are started directly from the $1^{1} \mathrm{~A}_{2}$ state (see S8 in SI). Finally, the transfer between $1^{3} \mathrm{~A}_{1} \rightarrow 1^{3} \mathrm{~A}_{2}$ is fast, estimated at $602 / 803 \mathrm{fs}$ for the forth and back transfers (in ethanol, this was estimated at $12 \mathrm{ps}) .{ }^{28}$ Solvents have a strong impact on the energetic order of $n \pi^{*}$ and $\pi \pi^{*}$ states of aromatic ketones, and this has a major impact on the dynamics in the excited state. Using the vacuum model, we have shifted the vertical energies to the values obtained for xanthone in solution (see Table S2 and Figure S6 in SI).

In polar solvents, the order of electronic states changes with respect to the gas phase. ${ }^{28,29}$ For triplets states, the ${ }^{3} \pi \pi^{*}$ is the lowest state both in water and ethanol. For singlet states, ethanol exhibits the same ordering as in gas phase. In contrast, in water the ${ }^{1} \mathrm{n} \pi^{*}$ fluctuates influenced by hydrogen bonds between water and carbonyl. From 1 ps $\mathrm{QM} / \mathrm{MD}$ water simulations, most snapshots exhibit an ${ }^{1} \mathrm{n} \pi^{*}$ state below the ${ }^{1} \pi \pi^{*}$ state (see Fig. S42 in SI). In such state ordering, quantum dynamics show that internal conversion ${ }^{1} \pi \pi^{*} \rightarrow{ }^{1} \mathrm{n} \pi^{*}$ is within 325 fs prior to the ISC in $2.11 \mathrm{ps}$ (see Fig. S43 in SI). The populated triplets are mostly the upper $2^{3} \pi \pi^{*}$ and $3^{3} \pi \pi^{*}$ at variance with the gas phase. In contrast, when ${ }^{1} \pi \pi^{*}$ is lowest, the wavepacket stays trapped in the singlets with a negligible ISC.

To summarize, we have developed a vibronic model for xanthone including Duschinsky effects for describing the internal conversion and ISC in xanthone. In gas phase, we determined an internal conversion of 85 fs from ${ }^{1} \pi \pi \rightarrow{ }^{1} n \pi^{*}$ states, followed by an ISC of 2.0 ps from ${ }^{1} n \pi^{*} \rightarrow{ }^{3} \pi \pi^{*}$. We find no evidence of direct transfer from ${ }^{1} \pi \pi^{*}$ to the triplets. In water, the reaction follows similar kinetics but the higher energy triplets are mainly populated. In conclusion, this dynamical model provides a complete description of xanthone and can be applied in the future to clarify the photochemistry of other aromatic ketones.

Acknowledgement MHR acknowledges financial support by the Agence Nationale pour la Recherche through the project BIOMAGNET (ANR-16CE29-0008-01). Centre de Calcul Intensif d'Aix-Marseille is acknowledged for granting access to its high performance computing resources. MAR and CdG acknowledge the Spanish ministry of Science and Innovation (Project PID2020-113187GB-I00) and the Genralitat de Catalunya (project 2017-SGR629) for financial support.

\section{Supporting Information Avail- able}

The supporting information is available containing the form and parameters of the model hamiltonian, the computational details for electronic structure and quantum dynamics simulations, the vibrationally resolved absorption spectrum and its interpretation, the time evolution of populations in gas phase and the first-order kinetic model behind, the 1D potential energy curves along each normal mode, the Cartesian coordiantes of the optimized structures in gas phase, the molecular dynamics simulations and the quantum dynamics in water.

\section{References}

(1) Kearns, D. R.; Case, W. A. Investigation of Singlet $\rightarrow$ Triplet Transitions by the Phosphorescence Excitation Method. III.
Aromatic Ketones and Aldehydes. J. Am. Chem. Soc. 1966 , 88, 5087-5097.

(2) Griffin, R. N. PHOSPHORESCENCE OF AROMATIC KETONES IN LOW-TEMPERATURE GLASSES Photochemistry and Photobiology 1968, 7, 159-173.

(3) Damschen, D. E.; Merritt, C. D.; Perry, D. L.; Scott, G. W. Talley, L. D. Intersystem crossing kinetics of aromatic ketones in the condensed phase. J. Phys. Chem. 1978, 82, 2268-2272.

(4) Scharf, G.; Winefordner, J. Phosphorescence characteristics of acetophenone, benzophenone, p-aminobenzophenone and michlercs ketone in various environments. Talanta 1986, 33, 17-25.

(5) El-Sayed, M. A. SpinâĂŤOrbit Coupling and the Radiationless Processes in Nitrogen Heterocyclics. J. Chem. Phys. 1963, 38, 2834-2838.

(6) Park, S. T.; Feenstra, J. S.; Zewail, A. H. Ultrafast electron diffraction: Excited state structures and chemistries of aromatic carbonyls. J. Chem. Phys. 2006, 124, 174707.

(7) Aloïse, S.; Ruckebusch, C.; Blanchet, L.; Réhault, J. Buntinx, G.; Huvenne, J.-P. The Benzophenone $\mathrm{S}_{1}\left(\mathrm{n}, \pi^{*}\right)$ $\rightarrow \mathrm{T}_{1}\left(\mathrm{n}, \pi^{*}\right)$ States Intersystem Crossing Reinvestigated by Ultrafast Absorption Spectroscopy and Multivariate Curve Resolution. J. Phys. Chem. A 2008, 112, 224-231.

(8) Kobayashi, T.; Nagakura, S. Picosecond time-resolved spectroscopy and the intersystem crossing rates of anthrone and fluorenone. Chem. Phys. Lett. 1976, 43, 429-434.

(9) Spighi, G.; Gaveau, M.-A.; Mestdagh, J.-M.; Poisson, L. Soep, B. Gas phase dynamics of triplet formation in benzophenone. Phys. Chem. Chem. Phys. 2014, 16, 9610-9618.

(10) Soep, B.; Mestdagh, J.-M.; Briant, M.; Gaveau, M.-A.; Poisson, L. Direct observation of slow intersystem crossing in an aromatic ketone, fluorenone. Phys. Chem. Chem. Phys. 2016 $18,22914-22920$.

(11) Chang, C.-W.; Sølling, T. I.; Diau, E. W.-G. Revisiting the photophysics of 9-fluorenone: Ultrafast time-resolved fluorescence and theoretical studies. Chem. Phys. Lett. 2017, 686, 218-222.

(12) Scaiano, J. C. Solvent effects in the photochemistry of xanthone. J. Am. Chem. Soc. 1980, 102, 7747-7753.

(13) Cavaleri, J. J.; Prater, K.; Bowman, R. M. An investigation of the solvent dependence on the ultrafast intersystem crossing kinetics of xanthone. Chem. Phys. Lett. 1996, 259, 495-502.

(14) Ley, C.; Morlet-Savary, F.; Fouassier, J.; Jacques, P. The spectral shape dependence of xanthone tripletâĂ on solvent polarity. J. Photochem. Photobiol. A 2000, 137, 8792.

(15) Ley, C.; Morlet-Savary, F.; Jacques, P.; Fouassier, J. Solvent dependence of the intersystem crossing kinetics of thioxanthone. Chemical Physics 2000, 255, 335-346.

(16) Ghosh, I.; Mukhopadhyay, A.; Koner, A. L.; Samanta, S. Nau, W. M.; Moorthy, J. N. Excited-state properties of fluorenones: influence of substituents, solvent and macrocyclic encapsulation. Phys. Chem. Chem. Phys. 2014, 16, 16436-16445.

(17) Ashpole, C. W.; Formosinho, S. J.; Porter, G. Pressure Dependence of Intersystem Crossing in Aromatic Vapours. Proceedings of the Royal Society of London. Series A, Mathematical and Physical Sciences 1971, 323, 11-28.

(18) Hill, N. S.; Coote, M. L. Internal Oriented Electric Fields as a Strategy for Selectively Modifying Photochemical Reactivity. J. Am. Chem. Soc. 2018, 140, 17800-17804.

(19) Rani, S.; Sobhanadri, J.; Prasada Rao, T. Solvent and concentration effects on the steady state fluorescence of fluorenone. J. Photochem. Photobiol. A 1996, 94, 1-5.

(20) Domcke, W., Yarkony, D., Koppel, H., Eds. Conical Intersections: Theory, Computation and experiment; Advanced Series in Physical Chemistry; World Scientific Publishing Co Pte Ltd, 2011; Vol. 17.

(21) Ohmori, N.; Suzuki, T.; Ito, M. Why does intersystem crossing occur in isolated molecules of benzaldehyde, acetophenone, and benzophenone? J. Phys. Chem. 1988, 92, 1086-1093.

(22) Rai-Constapel, V.; Etinski, M.; Marian, C. M. Photophysics of Xanthone: A Quantum Chemical Perusal. J. Phys. Chem. A 2013, 117, 3935-3944.

(23) Huix-Rotllant, M.; Burghardt, I.; Ferré, N. Population of triplet states in acetophenone: A quantum dynamics perspective. Comptes Rendus Chimie 2016, 19, 50-56.

(24) Favero, L.; Granucci, G.; Persico, M. Surface hopping investigation of benzophenone excited state dynamics. Phys. Chem. Chem. Phys. 2016, 18, 10499-10506.

(25) Rai-Constapel, V.; Kleinschmidt, M.; Salzmann, S.; SerranoAndr Ãl's, L.; Marian, C. M. Thioxanthone: on the shape of the first absorption band. Phys. Chem. Chem. Phys. 2010, 12, 9320-9327.

(26) Rai-Constapel, V.; Salzmann, S.; Marian, C. M. Isolated and Solvated Thioxanthone: A Photophysical Study. J. Phys. Chem. A 2011, 115, 8589-8596.

(27) $\bar{M}$ undt, $\overline{R . ;}$ Villnow, T.; Ziegenbein, C. T.; Gilch, P.; Marian, C.; Rai-Constapel, V. Thioxanthone in apolar solvents: ultrafast internal conversion precedes fast intersystem crossing. Phys. Chem. Chem. Phys. 2016, 18, 6637-6647.

(28) Satzger, H.; Schmidt, B.; Root, C.; Zinth, W.; Fierz, B. Krieger, F.; Kiefhaber, T.; Gilch, P. Ultrafast Quenching of the Xanthone Triplet by Energy Transfer: New Insight into the Intersystem Crossing Kinetics. J. Phys. Chem. A 2004, 108 $10072-10079$ 
(29) Heinz, B.; Schmidt, B.; Root, C.; Satzger, H.; Milota, F.; Fierz, B.; Kiefhaber, T.; Zinth, W.; Gilch, P. On the unusual fluorescence properties of xanthone in water. Phys. Chem. Chem. Phys. 2006, 8, 3432-3439.

(30) Heinz, B.; Schmidt, B.; Root, C.; Milota, F.; Fierz, B. Kiefhaber, T.; Zinth, W.; Gilch, P. Slow Fluorescence and Fast Intersystem Crossing - The Xanthone Anomaly. Ultrafast Phenomena XV. Berlin, Heidelberg, 2007; pp 513-515.

(31) Bracker, M.; Marian, C. M.; Kleinschmidt, M. Internal conversion of singlet and triplet states employing numerical DFT/MRCI derivative couplings: Implementation, tests, and application to xanthone. J. Chem. Phys. 2021, 155, 014102.

(32) Sharp, T. E.; Rosenstock, H. M. FranckâĂ ̌̌Condon Factors for Polyatomic Molecules. J. Chem. Phys. 1964, 41, 3453-3463.

(33) Baba, M.; Kamei, T.; Kiritani, M.; Yamauchi, S.; Hirota, N A phosphorescence study of jet-cooled xanthone. Chem. Phys. Lett. 1991, 185, 354-358.

(34) Ohshima, Y.; Fujii, T.; Fujita, T.; Inaba, D.; Baba, M. S ${ }^{1} \mathrm{~A}_{2}\left(\mathrm{n} \pi^{*}\right)$ and $\mathrm{S}_{2}{ }^{1} \mathrm{~A}_{1}\left(\pi \pi^{*}\right)$ States of Jet-Cooled Xanthone. J. Phys. Chem. A 2003, 107, 8851-8855. 


\section{Graphical TOC Entry}

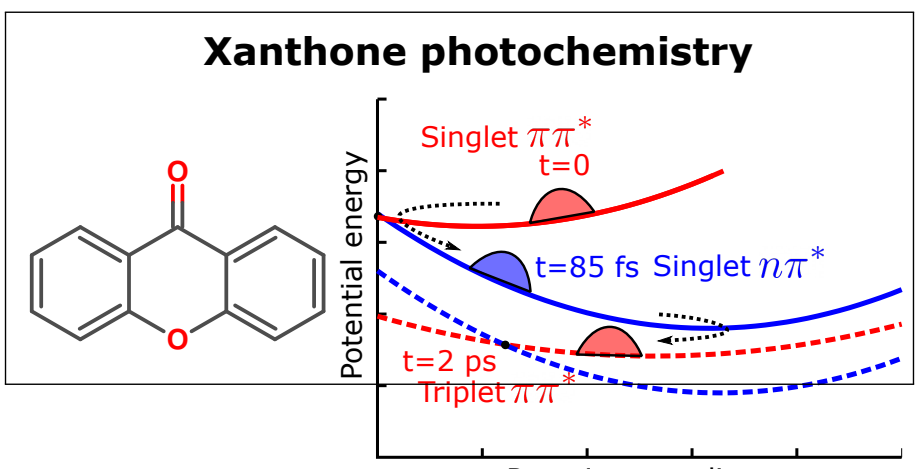

Reaction coordinate

Xanthone photochemistry has been determined from quantum dynamics to follow Kasha's rule and El-Sayed's rules. The origin of the ultrafast intersystem crossing is that the minimum of the lowest singlet is close to the crossing point with the triplet. 